Article

\title{
Impact of Insoluble Separation Layer Mechanical Properties on Disintegration and Dissolution Kinetics of Multilayer Tablets
}

\author{
Reiji Yokoyama ${ }^{1,2,3}$, Go Kimura $^{4}$, Jörg Huwyler ${ }^{1}$ (D), Ken-ichi Hosoya ${ }^{3}$ and Maxim Puchkov ${ }^{1, *(\mathbb{C})}$ \\ 1 Department of Pharmaceutical Sciences, Division of Pharmaceutical Technology, University of Basel, \\ Klingelbergstrasse 50, CH4056 Basel, Switzerland; reiji.yokoyama@shionogi.co.jp (R.Y.); \\ joerg.huwyler@unibas.ch (J.H.) \\ 2 Formulation R\&D Laboratory, CMC R\&D Division, SHIONOGI \& CO., LTD., Hyogo 660-0813, Japan \\ 3 Department of Pharmaceutics, Graduate School of Medicine and Pharmaceutical Sciences, \\ University of Toyama, Sugitani 2630, Toyama 930-0152, Japan; hosoyak@pha.u-toyama.ac.jp \\ 4 Production Technology Department, Manufacturing Division, Shionogi Pharma Co., LTD., \\ Hyogo 660-0813, Japan; go.kimura@shionogi.co.jp \\ * Correspondence: maxim.puchkov@unibas.ch; Tel.: +41-61-207-1619
}

Received: 21 February 2020; Accepted: 28 May 2020; Published: 29 May 2020

\begin{abstract}
Dissolution and disintegration of solid dosage forms such as multiple-layer tablet with different active ingredients depend on formulation and properties used in the formulations, and it may sometimes result in counterintuitive release kinetics. In this manuscript, we investigate the behavior of combined acetylsalicylic acid and mefenamic acid bi- and triple-layer formulations. We show that the simulation model with a cellular automata predicted the impact of the inert layer between the different active ingredients on each drug release and provide a good agreement with the experimental results. Also, it is shown that the analysis based on the Noyes-Whitney equation in combination with a cellular automata-supported dissolution and disintegration numerical solutions explain the nature of the unexpected effects. We conclude that the proposed simulation approach is valuable to predict the influence of material attributes and process parameters on drug release from multicomponent and multiple-layer pharmaceutical tablets and to help us develop the drug product formulation.
\end{abstract}

Keywords: disintegration simulation; poorly water-soluble drug; mefenamic acid; acetylsalicylic acid; Noyes-Whitney equation; cellular automata

\section{Introduction}

In the past decades, mostly fixed dose combination products (FDC) have been developed and introduced on the market [1]. In the development of FDC, the dosage form of the multi-layer tablets is selected since the multi-layer tablets have several advantages as compared to the monolithic conventional tablets. For example, one of the benefits of a multi-layer tablet geometries is a possibility to reduce chemical incompatibilities between active pharmaceutical ingredients (APIs) or between other formulation components by physical separation. Additional benefit of a multi-layer approach is a possibility to design dedicated release kinetics for each API in the dosage form $[2,3]$ to help prolonging the product's life cycle [4]. As an approach controlling and modifying the release rate of the API, the multilayer tablet technologies which combine the immediate release layers with the barrier or swellable/erodible properties was reported [5-7]. In those cases as well as in the case of multilayered pellet formulations, the drug release of the API was modified depending on the amount of hydrogel polymers such as hydroxy propyl methyl cellulose acetate succinate (HPMCAS) 
in the layer and the multi-layer design [8,9]. To understand the drug release mechanism from the experimental data set, kinetic mechanism studies with the mathematical fitting, for example, Higuchi model, Ritger-Peppas model and non-Fickian model, were reported [10,11]. However, it is challenging to predict the dissolution of the multi-layer tablets from the individual compressed layers of the same formulations as the layers in the multilayer tablets. As a reason, it is considered that there is a need for universal models reflecting the shape and other factors, e.g., components' colligative properties, and for real tablets or layered pellets which may affect the dissolution [12], despite numerous successful attempts of modeling individual shape effects, especially models based on parabolic partial differential equations and extensions of Noyes-Whitney models with fractal geometry [7,13-17].Therefore, there is a need to link dissolution with disintegration especially for the mentioned multilayer formulations with nontrivial geometries.

Over the past decades, numerous simulation models were developed in the field of pharmaceutical tablets compaction, dissolution, and disintegration [18-21], for example, models of mechanical and dissolution behavior of particles and tablets [19,22]. Numerical simulation models based on finite-element method (FEM) treats the powder as a continuous material and is used for simulation of the drug release from hydrogel-based matrix tablets [23]. In case of a significantly higher degree of formulation heterogeneity, the continuous methods are less applicable, especially for those systems where point contacts between formulation components play a significant role, and the use of discrete methods is appropriate [24-26]. It was reported that swelling behavior and the drug release from hydrophilic polymer tablets with different tablet shapes, components, and drug loading can be simulated with the discrete-element method (DEM) [24-27]. Besides DEM, the modeling technique with the cellular automata algorithm was proposed [28,29], and it was used to simulate the drug release of pharmaceutical tablets [30,31]. Importantly, three-dimensional cellular algorithms allow simulation of formulations containing different types of components organized as a large number of discrete cubic mesh; the simplicity of the calculation with the cellular automata enable the simulation with many different types of components, as compared to DEM models [32]. For example, it was reported in our previous work that the simulation algorithms could simulate the disintegration and dissolution of the mefenamic acid (MA) tablets very close to real experimental data [33]. In the simulation, the molecular dynamics simulation was adopted for the calculation of the diffusion coefficients of the API molecule. Other applications of the three-dimensional cellular automata algorithm include simulation of the disintegration time of pharmaceutical tablets [21] and of the buoyancy and drug release simulations of gastroretentive floating tablets [34,35].

From the advantages of the three-dimensional cellular automata, the purpose of the study was to simulate the dissolution of the multi-layer tablets with the three-dimensional cellular automata and to elucidate the impact of the insoluble layer on the formulation performance of the multi-layer tablets.

\section{Materials and Methods}

\subsection{Materials}

Mefenamic acid (SIGMA, St. Louis, MO, USA) and acetylsalicylic acid (ASA) (Lot: W004649, Glatt $\mathrm{GmbH}$, Binzen, Germany) were selected as a model compound to prepare rapidly disintegrating tablets. D-mannitol (Pearlitol 25C, Roquette, Lestrem, France) and microcrystalline cellulose (Avicel PH-101, FMC bioPolymer, Philadelphia, PA, USA) were used as diluents, and croscarmellose sodium (Ac-Di-Sol, FMC bioPolymer, Philadelphia, PA, USA) was used as a disintegrant. Hydroxypropyl cellulose (HPC SL, NIPPON SODA, Tokyo, Japan) was a binder, and magnesium stearate (Peter Greven GmbH \& Co, Bad Münstereifel, Germany) as a lubricant was also selected. Polycaprolactone (1639, Abifor, Zurich, Switzerland) was used for the inert 2nd layer of the triple-layer tablet. Cetyltrimethylammonium bromide (CTAB) (Merck, Kenilworth, NJ, USA) was used as a surfactant for dissolution tests. 


\subsection{Methods}

\subsubsection{Preparation of Tablets}

In this study, two single entities of ASA tablets and MA tablets were prepared. The formulation of the ASA tablets used for this study was presented in Table 1. For preparing the ASA tablet, ASA was granulated with the weighed excipients (i.e., D-mannitol, microcrystalline cellulose, croscarmellose sodium, and hydroxypropyl cellulose) in a high-shear mixer (MYCROMIX, OYSTAR Hüttlin, Schopfheim, Germany). The powders were mixed for $1 \mathrm{~min}$ at an impeller speed of $250 \mathrm{rpm}$ prior to the granulation process. Next, the granulation process was carried out at 250-rpm impeller speed/2000-rpm chopper speed. Hydroxypropyl cellulose aqueous solution $(10 \% w / w)$ was added at a spray rate of approximately $5 \mathrm{~g} / \mathrm{min}$. After completion of the binder solution, water was added at the same spray rate to flush the line. The process was continued for $1 \mathrm{~min}$. The obtained wet granules were dried and milled using a screen mill (Fitz mill model L1A, Fitz Patrick, Waterloo, ON, Canada). Afterward, the milled granules were weighed and mixed with croscarmellose sodium and magnesium stearate. The ASA tablets were prepared using a compaction simulator (StylOne, Medel pharma, Beynost, France) equipped with a tooling of $21.5 \times 10-\mathrm{mm}$ oval punch set. The setting value of the dwell times for pre-compression and main compression were $25 \mathrm{~ms}$. The compaction parameters are presented in Table 1. With respect to the MA tablets, the MA granulate and the tablet was prepared according to previous work [33].

Table 1. Formulation compositions and tablet compaction parameters. ASA: acetylsalicylic acid.

\begin{tabular}{ccc}
\hline \multirow{2}{*}{ Formulation Composition } & \multicolumn{2}{c}{ ASA Tablet } \\
\cline { 2 - 3 } & $\mathbf{m g}$ & $\mathbf{\% , w / w}$ \\
\hline Granule composition & 500.0 & 50.0 \\
ASA & 330.0 & 33.0 \\
D-mannitol & 100.0 & 10.0 \\
Microcrystalline cellulose & 20.0 & 2.0 \\
Croscarmellose sodium & 30.0 & 3.0 \\
Hydroxypropyl cellulose & 980.0 & 98.0 \\
Granulate & & \\
External phase composition & 10.0 & 1.0 \\
Croscarmellose sodium & 10.0 & 1.0 \\
Magnesium stearate & 1000.0 & - \\
Tablet weight & \multicolumn{2}{c}{7.1} \\
Tablet Parameters $(N=9)$ & \multicolumn{2}{c}{26} \\
Tablet porosity $(\%, v / v)$ & \multicolumn{2}{c}{} \\
Compressive force $(\mathrm{kN})$ &
\end{tabular}

The bi-layer tablet consists of MA and ASA layers, and the triple-layer tablet consists of MA, polycaprolactone, and ASA layers. The formulations of the bi-layer tablet and triple-layer tablet are shown in Table 2. The 1st layer, middle layer, and 3rd layer of the triple-layer tablet were ASA, polycaprolactone, and MA, respectively. The same ASA granulate and the MA granulate, which was used for each single layer tablet, were used in the multilayer tablets and compacted with the same tooling, which was used for single API tablets. The dwell times for pre-compression and main compression were set to $25 \mathrm{~ms}$. The compaction parameters are presented in Table 2.

\subsubsection{Determination of Tablet Porosity, Effective Compressive Stress, and Surface Area}

Mean tablet weight was evaluated with an electronic balance (AX204 Delta Range, Mettler Toledo, Greifensee, Switzerland). In addition, tablet diameter and tablet thickness were evaluated with a digital caliper (CD-15CPX, Mitutoyo, Kanagawa, Japan). All obtained values of tablet weight, diameter, and thickness were within $1 \%$ deviation. True densities of all raw materials were evaluated using 
helium pycnometry (AccuPyc 1330, Micrometrics, Norcross, GA, USA). The values of the ASA and polycaprolactone are $1.3928 \mathrm{~g} / \mathrm{cm}^{3}$ and $1.1600 \mathrm{~g} / \mathrm{cm}^{3}$, respectively. The values for other ingredients were reported previously [33].

Table 2. Formulation compositions and tablet compaction parameters. MA: mefenamic acid.

\begin{tabular}{|c|c|c|c|c|}
\hline \multirow{2}{*}{ Formulation Composition } & \multicolumn{2}{|c|}{ Bi-Layer Tablet } & \multicolumn{2}{|c|}{ Triple-Layer Tablet } \\
\hline & mg & $\%, w / w$ & mg & $\%, w / w$ \\
\hline \multicolumn{5}{|l|}{ Granule composition } \\
\hline ASA & 500.0 & 50.0 & 500.0 & 50.0 \\
\hline D-mannitol & 330.0 & 33.0 & 330.0 & 33.0 \\
\hline Microcrystalline cellulose & 100.0 & 10.0 & 100.0 & 10.0 \\
\hline Croscarmellose sodium & 20.0 & 2.0 & 20.0 & 2.0 \\
\hline Hydroxypropyl cellulose & 30.0 & 3.0 & 30.0 & 3.0 \\
\hline Granulate & 980.0 & 98.0 & 980.0 & 98.0 \\
\hline \multicolumn{5}{|l|}{ External phase composition } \\
\hline Croscarmellose sodium & 10.0 & 1.0 & 10.0 & 1.0 \\
\hline Magnesium stearate & 10.0 & 1.0 & 10.0 & 1.0 \\
\hline ASA layer weight & 1000.0 & - & 1000.0 & - \\
\hline $\begin{array}{c}\text { Separation layer } \\
\text { Polycaprolactone (PCL) }\end{array}$ & - & - & 300.0 & - \\
\hline \multicolumn{5}{|l|}{ Granule composition } \\
\hline $\mathrm{MA}^{1}$ & 250.0 & 50.0 & 250.0 & 50.0 \\
\hline D-mannitol & 165.0 & 33.0 & 165.0 & 33.0 \\
\hline Microcrystalline cellulose & 50.0 & 10.0 & 50.0 & 10.0 \\
\hline Croscarmellose sodium & 10.0 & 2.0 & 10.0 & 2.0 \\
\hline Hydroxypropyl cellulose & 15.0 & 3.0 & 15.0 & 3.0 \\
\hline Granulate & 490.0 & 98.0 & 490.0 & 98.0 \\
\hline \multicolumn{5}{|l|}{ External phase composition } \\
\hline Croscarmellose sodium & 5.0 & 1.0 & 5.0 & 1.0 \\
\hline Magnesium stearate & 5.0 & 1.0 & 5.0 & 1.0 \\
\hline MA layer weight & 500.0 & - & 500.0 & - \\
\hline Tablet weight & 1500.0 & - & 1800.0 & - \\
\hline \multirow{2}{*}{ Tablet porosity $(\%, v / v)$} & \multirow{2}{*}{\multicolumn{2}{|c|}{7.4 (ASA layer) }} & \multicolumn{2}{|c|}{7.4 (ASA layer) } \\
\hline & & & \multicolumn{2}{|c|}{11.1 (MA layer) } \\
\hline Compressive force $(\mathrm{kN})$ & \multicolumn{2}{|c|}{21} & \multicolumn{2}{|c|}{21} \\
\hline
\end{tabular}

The true density of each layer in the tablets was calculated according to Equation (1).

$$
\rho_{\text {layer }}=\frac{1}{\sum_{i=1}^{n} \frac{X_{i}}{\rho_{i}}}
$$

where $\rho_{\text {layer }}$ and $\rho_{i}$ are the true densities $\left(\mathrm{g} / \mathrm{cm}^{3}\right)$ of the layer and each raw material in the layer, respectively, and $X_{i}$ is the weight fraction of each component.

The porosity $\varepsilon$ of the layers was determined according to Equation (2).

$$
\varepsilon=1-\frac{m}{V \rho_{\text {layer }}}
$$

where $V$ is the layer volume calculated from the experimental layer thickness and F-CAD software and where $\mathrm{m}$ is the weight of the layer. 
The horizontal cross-sectional area and the surface area of the tablets were calculated using the computer-aided design software T-CAD (T-CAD, Tokyo, Japan), and the effective compressive stress $P_{\text {comp }}(\mathrm{MPa})$ was calculated according to Equation (3).

$$
P_{\text {comp }}=\frac{F_{\text {comp }} \times 1000}{S_{\text {cross section }}}
$$

where $S_{\text {cross section }}\left(\mathrm{mm}^{2}\right)$ is the horizontal cross section area of the tablets and $F_{\text {comp }}(\mathrm{kN})$ is the resultant compressive force in the compression of the tablets.

\subsubsection{Disintegration Test}

The disintegration times were measured using a disintegration tester (Sotax DT3, Sotax AG, Allschwil, Switzerland), according to the United States Pharmacopeia (USP) 24 method. Tests were carried out in $900 \mathrm{~mL}$ of $50 \mathrm{mM}$ sodium phosphate buffer $\left(\mathrm{pH}\right.$ 6.8) containing $1 \% \mathrm{CTAB}$ at $37^{\circ} \mathrm{C} \pm 0.5^{\circ} \mathrm{C}$ $(n=3)$. All tests were done in triplicate using six tablets for each test.

\subsubsection{Dissolution Test}

Dissolution tests of the ASA tablets, the bilayer tablets, and the triple layer tablets were carried out using the USP dissolution apparatus II with a paddle rotation of $75 \mathrm{rpm}(n=6)$ (AT7smart, Sotax, Allschwil, Switzerland). The dissolution media and its volume is $900 \mathrm{~mL}$ of $50 \mathrm{mM}$ sodium phosphate buffer ( $\mathrm{pH}$ 6.8) containing $1 \% \mathrm{CTAB}$ at $37^{\circ} \mathrm{C} \pm 0.5^{\circ} \mathrm{C}$. Drug rate were analyzed by using an HPLC system (Agilent 1100 LC, Santa Clara, CA, United States) equipped with a reverse phase T3 Atlantis C18 column ( 3 um, $3.0 \times 20 \mathrm{~mm}$, Waters, MA, USA) every $5 \mathrm{~min}$. The wavelength of the UV detector was set to $230 \mathrm{~nm}$ and $237 \mathrm{~nm}$ for MA and ASA, respectively. The column temperature was maintained at $40{ }^{\circ} \mathrm{C}$. The mobile phase used for MA and ASA was acetonitrile/10 $\mathrm{mM}$ ammonium formate buffer solution ( $\mathrm{pH}$ 3.5). The bilayer tablet and triple layer tablet were inserted into the dissolution vessels to ensure that the MA layer is the upper side in the bottom of the vessels. (to avoid the variation of dissolution due to difference in the layer position)

\subsubsection{Water Sorption Test}

Liquid sorption was measured for MA and ASA granulates in order to compare liquid imbibition rates of both layer components. Measurements were carried out with Krüss Tensiometer K100 (Krüss GmbH, Hamburg, Germany), and data were analyzed with Laboratory Desktop, Ver. 3.2.2.3068 (Krüss GmbH, Germany). The weight of the granulates used for experiments was approx. $700 \mathrm{mg}$; the inner diameter of the measurement cell was $11.33 \mathrm{~mm}$. The liquid medium was distilled water. Time for liquid uptake experiment was taken enough to reach a plateau region, which indicates no significant further weight increase. The slope and capillary constants were calculated with the Laboratory Desktop software after manual selection of linear sections on the squared mass vs. time plots. Every sorption experiment was carried out in triplicates.

\subsection{Simulation of Drug Release with Cellular Automata}

The software package F-CAD v.2.0 was used for the simulations of the drug release of ASA tablets, the bilayer tablets, and the triple-layer tablets. For the simulation of the experimental tablet, the virtual tablets created in the software, for which geometrical information (i.e., size, shape, and layer order) is identical to the experimental tablets (i.e., $21.5 \times 10-\mathrm{mm}$ oval tablet), were generated. The virtual tablet was discretized into a cubic grid using a voxel side length of $74 \mu \mathrm{m}$ (with $293^{3}$ elements). In our previous work [33], the molecular dynamics approach was adopted to calculate the simulation constants of MA particle, which expresses the dissolution rate in the simulation model with a cellular automata; thus, the same method of the molecular dynamics approach was adopted for the calculation of ASA simulation constants as well. 
Comparison of Drug Release Pattern between Experimental and Simulated Profiles

To calculate the similarity factor $\left(f_{2}\right)$ of the drug release between experimental data and simulated data, Equation (4) was used [36].

$$
f_{2}=50 \times \log \left\{\left[1+\frac{1}{n} \sum_{i=1}^{n}\left(R_{t}-T_{t}\right)^{2}\right]^{-0.5} \times 100\right\}
$$

where $n$ is the number of time points, $R_{t}$ is the dissolution rate of the experimental tablet at time $t$, and $T_{t}$ is the dissolution rate of the simulated tablet at time $t$. A similarity factor $\left(f_{2}\right)$ greater than 50 indicates a close correlation between simulated and experimental data.

\subsection{Fitting of Noyes-Whitney Dissolution Model with Experimental Data}

Fitting of the experimental dissolution results were made with Mathematica 11.0 (Wolfram Research Inc., Champaign, IL, USA). The screenshot is introduced in Supplementary Figure S1. The source code is available in the supplement. The data were fitted with the Noyes-Whitney equation:

$$
\frac{\mathrm{d} C}{\mathrm{~d} t}=\frac{D S}{h}\left(C_{s}-C\right)
$$

where $C$ is the fraction of released $\operatorname{drug}(\%, w / v), C s$ is the drug solubility $(\%, w / v), D$ is the diffusivity coefficient $\left(\mathrm{cm}^{2} / \mathrm{s}\right), h$ is the length of the unstirred layer $(\mathrm{cm})$, and $S$ is the specific surface of the sample $\left(\mathrm{cm}^{2} / \mathrm{g}\right)$.

Unlike the dissolution and disintegration simulations with three-dimensional cellular automata, the model of Noyes-Whitney does not consider the spatial differentials and material fluxes, which mainly govern the dynamics of dissolution and disintegration of the tablets. The main use and purpose of the Noyes-Whitney equation in this manuscript was to obtain a single coefficient fitting model for comparative analysis of the experimental data. Specific surface was the sole fitting coefficient selected for this purpose. Fittings were made for release data of ASA layer for both triple- and bi-layer tablets. The obtained values for $S$ were interpreted as surface available for dissolution contact in both tablet geometries.

\section{Results}

\subsection{In Vitro Evaluation of Drug Release}

The properties of the experimental tablets and their compaction condition are summarized in Table 3. Also, with respect to the effective surface area contributing the disintegration and dissolution of the tablets in the dissolution tests, it is considered in the triple-layer tablet that the effective surface area for MA layer and the ASA layer decreased by the contacting polycaprolactone layer, which does not penetrate the water into these adjacent layers. Based on the theory, the effective surface area was calculated and given in Table 4.

Table 3. Tablet properties and compaction conditions.

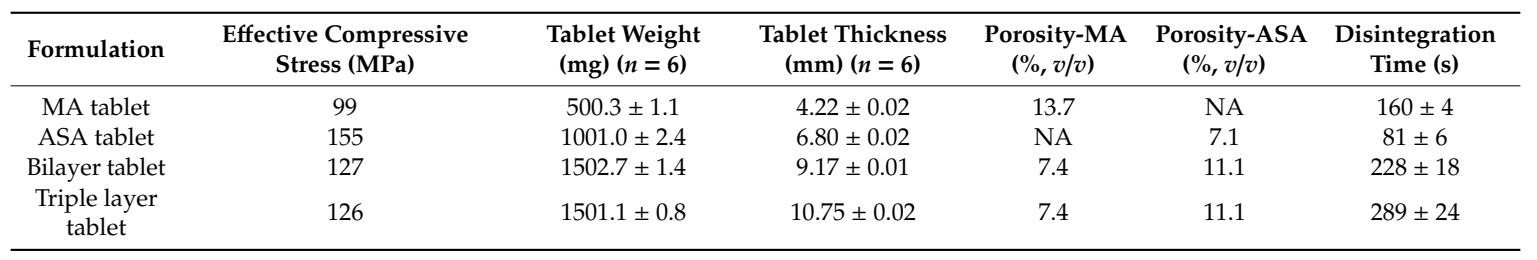


Table 4. Comparison of tablets' surface area and areas of individual layers.

\begin{tabular}{ccccccc}
\hline \multirow{2}{*}{ Item } & MA Tablet & ASA Tablet & \multicolumn{2}{c}{ Bilayer Tablet } & \multicolumn{2}{c}{ Triple-Layer Tablet } \\
\cline { 5 - 7 } & & MA Layer & ASA Layer & MA Layer & ASA Layer \\
\hline $\begin{array}{c}\text { Surface area } \\
\left(\mathrm{mm}^{2}\right)\end{array}$ & 350.2 & 484.4 & 289.3 & 318.4 & 289.3 & 318.4 \\
$\%$ of Control & MA Control & ASA Control & $83 \%$ & $66 \%$ & $83 \%$ & $66 \%$ \\
\hline
\end{tabular}

In vitro drug release testing of the MA tablets, the ASA tablets, the bilayer tablets, and the triple-layer tablets were carried out, and the results of MA and ASA are shown in Figures 1 and 2 , respectively.

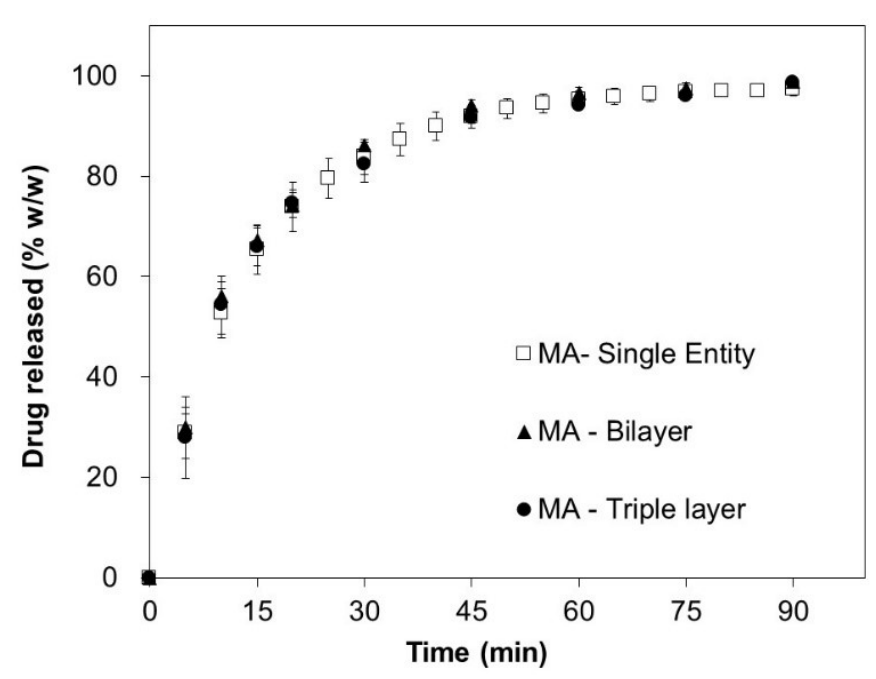

Figure 1. Experimental drug release of mefenamic acid (MA).

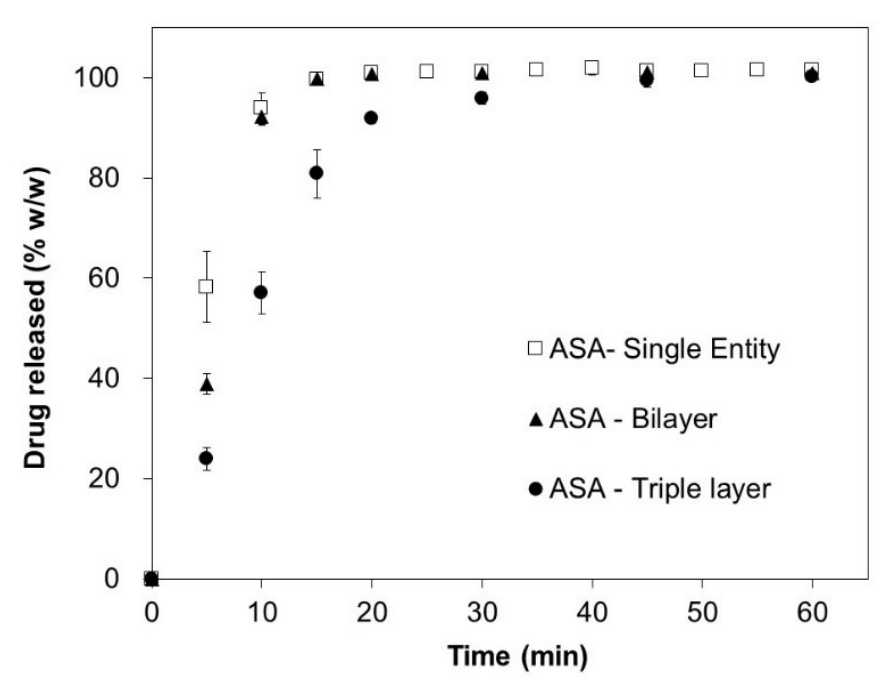

Figure 2. Experimental drug release of acetylsalicylic acid (ASA).

The obtained release profiles for MA in Figure 1 are identical for all study cases, i.e., single tablet, bilayer tablet, and triple layer tablet. An unexpectedly different result is seen for ASA (Figure 2), with significantly slower release rates in the case of a triple-layer tablet formulation. As shown in Table 3, the disintegration time of the bilayer tablet and the triple-layer tablet are longer than that of the single component tablets, corresponding to the rank-order of the in vitro dissolution data.

Results of the fitting of the experimental data with differential form of the Noyes-Whitney equation yields 1.8 times reduction in contact surface for ASA in triple-layered tablet $\left(R^{2}=0.99\right)$. 
The results of the liquid uptake measurements for the granulates used to compact the layer of bi- and triple-layered tablet are show in Table 5. The total amount of the adsorbed liquid for both granulates was not significantly different. On the other hand, the rates of the liquid imbibition, i.e., a slope and the capillary constant, show differences by factors of approx. 6 and 4, respectively.

Table 5. Results of the liquid uptake measurements for MA and ASA granulates $(n=3$, error range is given as standard deviation).

\begin{tabular}{cccc}
\hline & Maximum Liquid Uptake, $\mathbf{\%}(\mathbf{w} / \mathbf{w})$ & Slope, $\mathbf{g}^{\mathbf{2}} / \mathbf{s}$ & Capillary Constant $\times \mathbf{1 0}^{\mathbf{- 6}}$ \\
\hline MA & $78 \pm 3.5$ & $0.013 \pm 4.5 \times 10^{-3}$ & $1.81 \pm 0.63$ \\
ASA & $83 \pm 5.7$ & $0.064 \pm 1.4 \times 10^{-2}$ & $8.84 \pm 1.94$ \\
\hline
\end{tabular}

\subsection{Comparison of in Silico Drug Release Profiles between Bilayer Tablet and Triple-Layer Tablet}

The in silico drug release of MA and ASA from the bilayer tablets and the triple layer tablets, which was simulated with cellular automata-based numeric models, are shown in Figure 3, respectively. Also, the comparison between in silico and in vitro release profiles from the bilayer tablets and triple-layer tablets are shown in Figures 4 and 5, respectively.

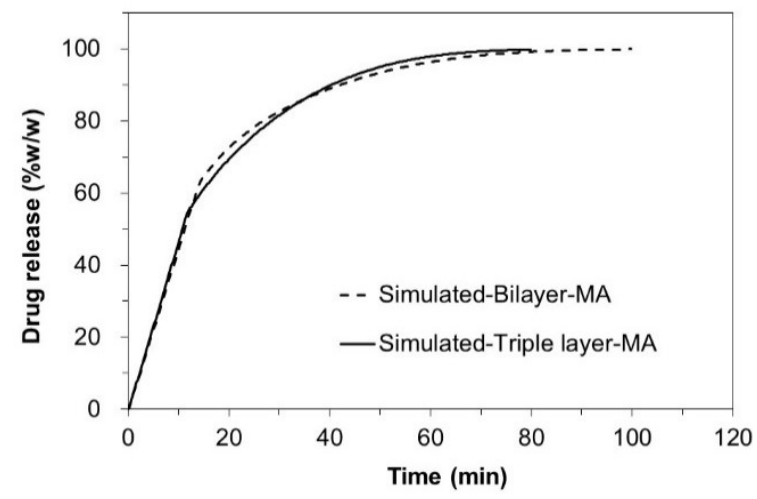

(a)

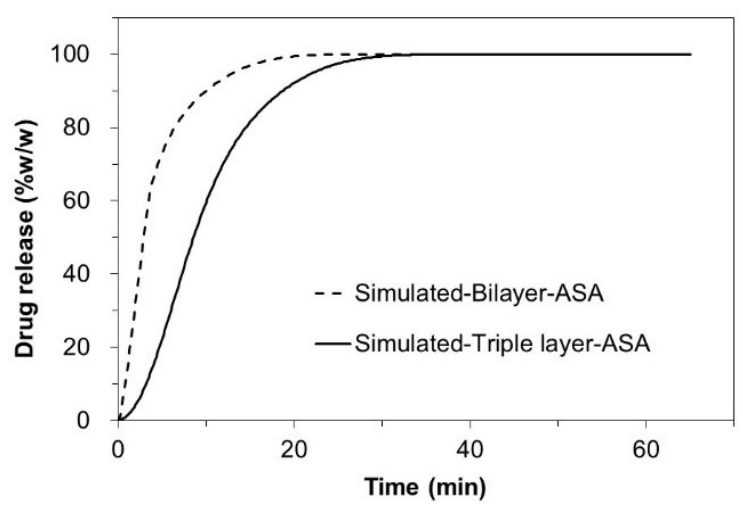

(b)

Figure 3. Comparison of in silico release profiles between the bilayer tablets (dotted lines) and the triple-layer tablets (solid lines) (a) MA; (b): ASA.

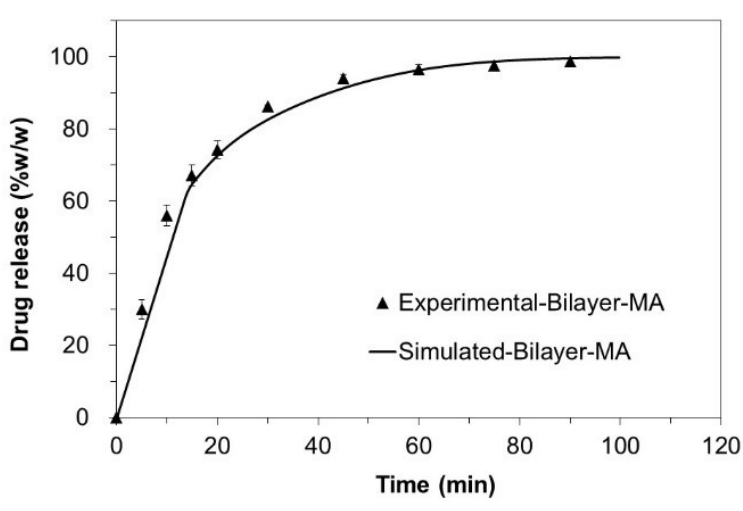

(a)

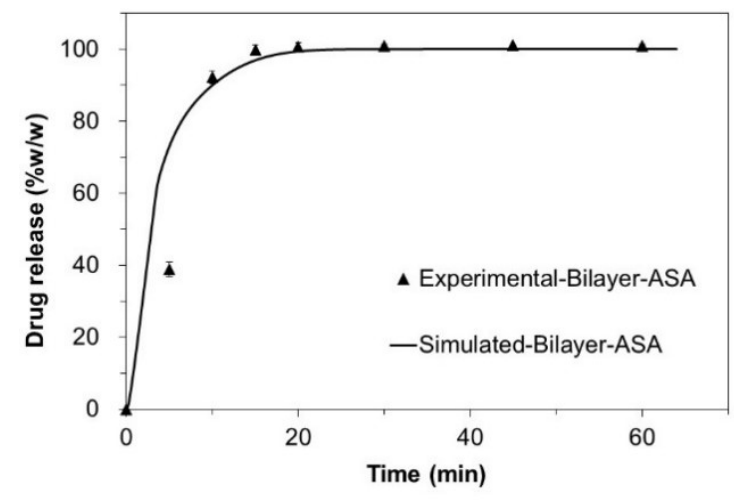

(b)

Figure 4. Comparison between in silico (solid lines) and in vitro (filled triangle) release profiles from the bilayer tablets (a) MA; (b) ASA. 


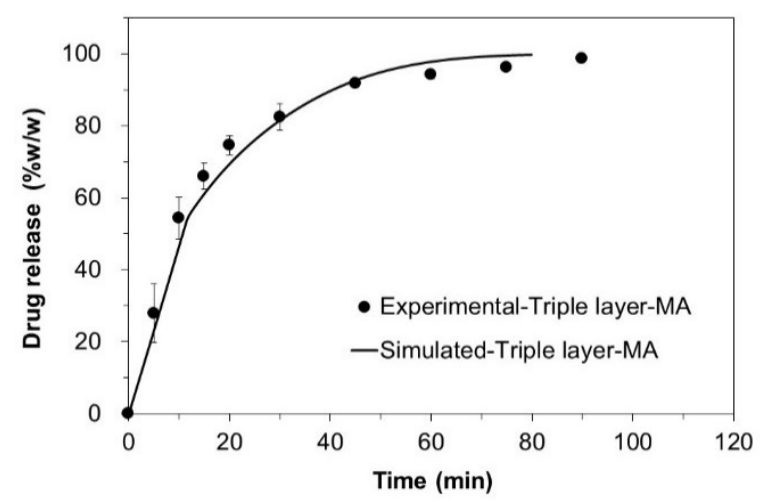

(a)

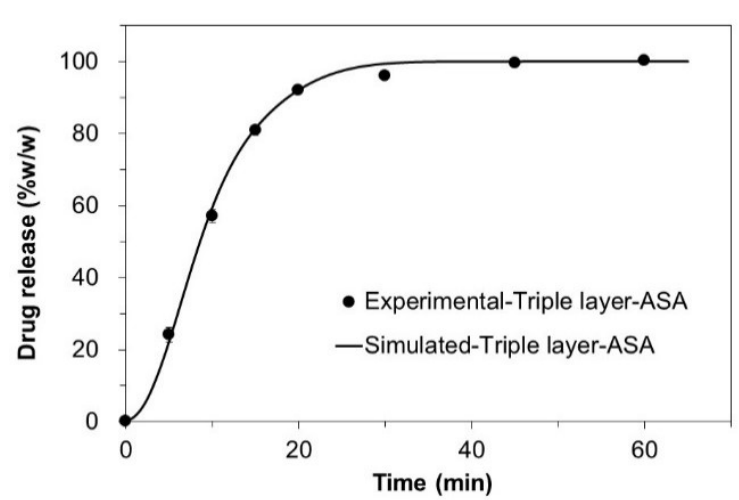

(b)

Figure 5. Comparison between in silico (solid lines) and in vitro (filled circles) release profiles from the triple layer tablets (a) MA; (b) ASA.

An attempt to simulate the dissolution profiles of individual layers and the tablet as a whole resulted in the simulated curves of high similarity to the experimentally obtained data, therefore, suggesting similarity between real physical processes and their simulated counterparts. In Table 6, the corresponding $f_{2}$ factors are given as a measure of similarity between simulated and experimental release profiles of MA and ASA bi- and triple-layered tablets.

Table 6. Summary of similarity factors $\left(f_{2}\right)$ between in vitro and in silico drug release profiles.

\begin{tabular}{cccc}
\hline \multicolumn{2}{c}{ Tablet } & MA & ASA \\
\hline \multirow{2}{*}{ Similarity factor $\left(f_{2}\right)$} & Bilayer tablets & 57 & 86 \\
& Triple layer tablets & 75 & 87 \\
\hline
\end{tabular}

\section{Discussion}

The experimental results shown in Figures 1 and 2 were not expected in the case of ASA triple-layered tablet. The release profiles suggest significant reduction in the release rate for highly soluble compound as compared to the unchanged release rate of the low-soluble MA component. The change is only observed if an inert layer of the polycaprolactone is introduced between layers, i.e., in the statistical analysis (one-way ANOVA) of dissolution rates at $10 \mathrm{~min}, 15 \mathrm{~min}$, and $30 \mathrm{~min}$, $p$-value $<0.05$ is only observed when comparing the dissolution rates between ASA tablets and triple-layer tablets. It is important to say that this effect cannot be simply explained by a geometrical contact surface reduction for ASA layer through MA or the inert separation layer as, in such a case, a similar effect would be observed for bi-layer tablets, i.e., tablets without separation component. On the contrary, the same behavior cannot be repeated for identically layered tablet composition without an intermediate separation. The results of fitting analysis suggest that ASA layer in a triple-layered tablet has reduced contact surface (by 1.8 times, according to Noyes-Whitney model-based analysis) exposed towards the dissolution medium. At the same time, the ASA layer is a highly soluble formulation and should have shown little or no dependency on the drug release from the MA layer. The results of the liquid uptake measurements corroborate the latter assumption. As shown in Table 5, the rate of liquid uptake for ASA layer is approx. 6 times faster than the capillary uplift registered for MA granules. The analysis of the simulated and experimental data of a bi-layer tablets, i.e., the tablets without inert separation layer, confirms that the upper layer with MA formulation is disintegrated faster due to its reduced thickness as compared to ASA layer and, hence, introduces no more obstacle for a liquid front to ingress into ASA layer. The Polycaprolactone (PCL) material apparently blocks this action for triple-layered tablets, as it remains attached to the surface of the ASA layer long after complete disintegration of the MA layer. The sensitivity of the ASA formulation to the geometry of the 
tablet was not expected, as it is normally not observed for fast disintegrating immediate release tablet formulations with highly soluble active substances. However, neither the three-dimensional cellular algorithms simulation of the drug release nor fitting of experimental data with the Noyes-Whitney model suggest an alternative explanation of the role of the inert layer. Similar findings reported in Reference [37] were also explained by different surface-to-volume ratios for single- and multi-layer formulations. The disintegration mechanism of ASA layer is different to the one of MA layer and can be best characterized as being erosion-driven. The difference in the disintegration mechanisms for almost identical formulations, i.e., not taking into account the geometrical differences, can be best explained by different diffusivities and aqueous solubilities of the active substances. Interestingly, the visual observation of the tablets' behavior during dissolution suggests rather limited liquid imbibition despite sufficient porosity; liquid transport in the porous meshwork is, apparently, not intensive enough to cause an immediate disintegration; therefore, an erosion-driven disintegration occurs. An effect of porous transport passivation may be caused by an increased viscosity of intra-porous liquid due to dissolved tablet components, such as ASA and mannitol. On the other hand, porosity of the ASA layer was lower as compared to MA (Table 3), which would allow only limited liquid imbibition into tablet body. In order to increase liquid sorption rate and, therefore, to induce disintegration and consequently dissolution speed, the porosity of the pharmaceutical compacts should be greater than the critical value, known as a percolation threshold [38]. However, the higher porosities of tablets lead to reduced tensile strength of the compact; therefore, such an approach to increase liquid diffusivity should be taken with care.

Another important aspect which was identified in this study is suitability of the two dissolution models. The Noyes-Whitney model, which accounts for basic characteristics of dissolving solid, e.g., diffusion and solubility parameters, can be applied for general description of the dissolution behavior under the assumption that the dissolving solid is a homogeneous solid. Significantly better modeling results can be obtained with models based on numerical methods, such as cellular automata method, used in this study. In the left side of Figure 5, the experimental release data of ASA are modelled with cellular automata (solid line). It is possible to spot a slightly sigmoidal shape of the modelled curve generated by the simulation. The models based solely on the Noyes-Whitney kinetic will not produce similar curves without significant extension of the model equations and introduction of further assumptions or simplifications.

The results of the study suggest that, for practical design of the multiple layer tablets, the intermediate layer must be designed as either a soluble or fast disintegrating component to avoid reduction of the dissolution rate, unless otherwise desired.

Inert layer material selection is not a trivial task, especially if this material must be compressed into fast disintegrating layers during tablet production. The layer must maintain separation integrity in order to effectively separate the active ingredients for enhanced stability of the final medicinal product.

\section{Conclusions}

In this study, the drug release from triple-layer multicomponent tablet formulation of mefenamic and acetylsalicylic acids was evaluated in vitro and in silico. Numerical simulations were applied to reveal the role of inert separation layer on the changing release behavior of the bi- and tri-layer tablets. For in silico simulation, the model with the three-dimensional cellular automata algorithm showed good agreements with the experimental dissolution data and predicted the impact of the insoluble layer on the dissolution of the APIs in both layers. The unmodified Noyes-Whitney dissolution model does not take disintegration into account and, therefore, was used only as a fitting model for comparative purposes. It is concluded that the simulation based on numerical methods, such as cellular automata, can help to understand the influence of different materials and their attributes, geometry, and process parameters on drug release from multicomponent and multiple-layer pharmaceutical tablet and can aid in rapid drug product formulation. 
The results of this study identify the impact of the insoluble layer on the formulation performance of multiple-layer tablets. Introduction of the insoluble and inert layers may significantly reduce the dissolution speed due to reduced surface of both layers. This effect is present even if the bi-layer tablets composed of the same materials do not show dissolution rate reduction. This often-undesired effect will disappear if the separation layer can be composed of either fast soluble or fast disintegrating material.

Supplementary Materials: The following are available online at http://www.mdpi.com/1999-4923/12/6/495/s1, Figure S1. Screenshot of Wolfram Mathematica.

Author Contributions: Conceptualization, R.Y. and G.K.; methodology, R.Y. and M.P.; study concept and simulation software, M.P.; data curation, R.Y. and M.P.; writing—original draft preparation, R.Y.; writing-review and editing, M.P., J.H. and K.-i.H. All authors have read and agreed to the published version of the manuscript.

Funding: This research received no external funding.

Acknowledgments: The authors thank Shionogi \& Co., LTD. for the research opportunity.

Conflicts of Interest: The authors declare no conflicts of interest. Reiji Yokoyama is the employee of SHIONOGI \& CO., LTD. Go Kimura is employee of Shionogi Pharma Co., LTD. The companies had no role in the design of the study; in the collection, analyses, or interpretation of data; in the writing of the manuscript; and in the decision to publish the results.

\section{References}

1. Desai, D.; Wang, J.; Wen, H.; Li, X.; Timmins, P. Formulation design, challenges, and development considerations for fixed dose combination (FDC) of oral solid dosage forms. Pharm. Dev. Technol. 2013, 18, 1265-1276. [CrossRef] [PubMed]

2. Nirmal, J.; Saisivam, S.; Peddanna, C.; Muralidharan, S.; Godwinkumar, S.; Nagarajan, M. Bilayer tablets of atorvastatin calcium and nicotinic acid: Formulation and evaluation. Chem. Pharm. Bull. 2008, 56, 1455-1458. [CrossRef] [PubMed]

3. Shiyani, B.; Gattani, S.; Surana, S. Formulation and Evaluation of Bi-layer Tablet of Metoclopramide Hydrochloride and Ibuprofen. AAPS PharmSciTech 2008, 9, 818-827. [CrossRef] [PubMed]

4. Qiu, Y.; Chen, Y.; Zhang, G.; Liu, L.; Porter, W. Developing Solid Oral Dosage Forms. Pharmaceutical Theory and Practice. Libro; Academic Press: Cambridge, MA, USA, 2009; ISBN 9780444532428.

5. Efentakis, M.; Peponaki, C. Formulation study and evaluation of matrix and three-layer tablet sustained drug delivery systems based on carbopols with isosorbite mononitrate. AAPS PharmSciTech 2008, 9, 917-923. [CrossRef] [PubMed]

6. Phaechamud, T. Variables influencing drug release from layered matrix system comprising hydroxypropyl methylcellulose. AAPS PharmSciTech 2008, 9, 668-674. [CrossRef] [PubMed]

7. Abdul, S.; Poddar, S. A flexible technology for modified release of drugs: Multi layered tablets. J. Control. Release 2004, 97, 393-405. [CrossRef]

8. Kállai-Szabó, N.; Luhn, O.; Bernard, J.; Kállai-Szabó, B.; Zelkó, R.; Antal, I. Comparative dissolution study of drug and inert isomalt based core material from layered pellets. J. Pharm. Biomed. Anal. 2014, 98, 339-344. [CrossRef]

9. Wei, H.E.; An, L.F.; Qing, D.U.; Iang, B.X.; Chun-lei, L.I.; Ai, M.B.; Hang, Y.C.; Ao, D.C. Design and in Vitro/in Vivo Evaluation of Multi-layer Film Coated Pellets for Omeprazole. Chem. Pharm. Bull. 2009, 57, 122-128.

10. Van Nguyen, H.; Nguyen, V.H.; Lee, B.J. Dual release and molecular mechanism of bilayered aceclofenac tablet using polymer mixture. Int. J. Pharm. 2016, 515, 233-244. [CrossRef]

11. Fu, Q.; Su, X.; Hou, Y.; Li, M.; Li, J.; Sun, J.; He, Z. Once-daily amoxicillin immediate- and extended-release bilayer tablets. Powder Technol. 2016, 301, 405-411. [CrossRef]

12. Kállai, N.; Luhn, O.; Dredán, J.; Kovács, K.; Lengyel, M.; Antal, I. Evaluation of Drug Release From Coated Pellets Based on Isomalt, Sugar, and Microcrystalline Cellulose Inert Cores. AAPS PharmSciTech 2010, 11, 383-391. [CrossRef] [PubMed]

13. Mikac, U.; Demsar, A.; Demsar, F.; Serša, I. A study of tablet dissolution by magnetic resonance electric current density imaging. J. Magn. Reson. 2007, 185, 103-109. [CrossRef] [PubMed] 
14. Reynolds, T.D.; Mitchell, S.A.; Balwinski, K.M. Investigation of the effect of tablet surface area/volume on drug release from hydroxypropylmethylcellulose controlled-release matrix tablets. Drug Dev. Ind. Pharm. 2002, 28, 457-466. [CrossRef] [PubMed]

15. Farin, D.; Avnir, D. Use of fractal geometry to determine effects of surface morphology on drug dissolution. J. Pharm. Sci. 1992, 81, 54-57. [CrossRef] [PubMed]

16. Cooney, D.O. Effect of geometry on the dissolution of pharmaceutical tablets and other solids: Surface detachment kinetics controlling. AIChE J. 1972, 18, 446-449.

17. Rippie, E.G.; Johnson, J.R. Regulation of dissolution rate by pellet geometry. J. Pharm. Sci. 1969, 58, $428-431$. [CrossRef]

18. Lao, L.L.; Peppas, N.A.; Boey, F.Y.C.; Venkatraman, S.S. Modeling of drug release from bulk-degrading polymers. Int. J. Pharm. 2011, 418, 28-41. [CrossRef]

19. Siepmann, J.; Siepmann, F. Mathematical modeling of drug dissolution. Int. J. Pharm. 2013, 453, 12-24. [CrossRef]

20. Frenning, G. An efficient finite/discrete element procedure for simulating compression of 3D particle assemblies. Comput. Methods Appl. Mech. Eng. 2008, 197, 4266-4272. [CrossRef]

21. Kimura, G.; Puchkov, M.; Leuenberger, H. An attempt to calculate in silico disintegration time of tablets containing mefenamic acid, a low water-soluble drug. J. Pharm. Sci. 2013, 102, 2166-2178. [CrossRef]

22. Macheras, P.; Iliadis, A. Modeling in Biopharmaceutics, Pharmacokinetics, and Pharmacodynamics; Interdisciplinary Applied Mathematics; Springer: New York, NY, USA, 2006; Volume 30, ISBN 0-387-28178-9.

23. Lamberti, G.; Galdi, I.; Barba, A.A. Controlled release from hydrogel-based solid matrices. A model accounting for water up-take, swelling and erosion. Int. J. Pharm. 2011, 407, 78-86. [CrossRef] [PubMed]

24. Kimber, J.A.; Kazarian, S.G.; Štěpánek, F. Modelling of pharmaceutical tablet swelling and dissolution using discrete element method. Chem. Eng. Sci. 2012, 69, 394-403. [CrossRef]

25. Kimber, J.A.; Kazarian, S.G.; Štěpánek, F. DEM simulation of drug release from structurally heterogeneous swelling tablets. Powder Technol. 2013, 248, 68-76. [CrossRef]

26. Kimber, J.A.; Kazarian, S.G.; Štěpánek, F. Formulation design space analysis for drug release from swelling polymer tablets. Powder Technol. 2013, 236, 179-187. [CrossRef]

27. Kimber, J.A.; Kazarian, S.G.; Štěpánek, F. Microstructure-based mathematical modelling and spectroscopic imaging of tablet dissolution. Comput. Chem. Eng. 2011, 35, 1328-1339. [CrossRef]

28. Rybacki, S.; Himmelspach, J.; Uhrmacher, A.M. Experiments with Single Core, Multi-core, and GPU Based Computation of Cellular Automata. In Proceedings of the First International Conference on Advances in System Simulation, Porto, Portugal, 20-25 September 2009; pp. 62-67. [CrossRef]

29. Harvey, A. A New Kind of Science; Wolfram Media: Somerville, MA, USA, 2002; Volume 30, ISBN 1-579-55008-8.

30. Puchkov, M.; Tschirky, D.; Leuenberger, H. 3-D cellular automata in computer-aided design of pharmaceutical formulations: Mathematical concept and F-CAD software. In Formulation Tools for Pharmaceutical Development; Elsevier B.V.: Amsterdam, The Netherlands, 2013; pp. 155-201. ISBN 9781907568992.

31. Laaksonen, H.; Hirvonen, J.; Laaksonen, T. Cellular automata model for swelling-controlled drug release. Int. J. Pharm. 2009, 380, 25-32. [CrossRef]

32. Ketterhagen, W.R.; AM Ende, M.T.; Hancock, B.C. Process modeling in the pharmaceutical industry using the discrete element method. J. Pharm. Sci. 2009, 98, 442-470. [CrossRef]

33. Yokoyama, R.; Kimura, G.; Schlepütz, C.M.; Huwyler, J.; Puchkov, M. Modeling of disintegration and dissolution behavior of mefenamic acid formulation using numeric solution of noyes-whitney equation with cellular automata on microtomographic and algorithmically generated surfaces. Pharmaceutics 2018, 10, 259. [CrossRef]

34. Eberle, V.A.; Schoelkopf, J.; Gane, P.A.C.; Alles, R.; Huwyler, J.; Puchkov, M. Floating gastroretentive drug delivery systems:Comparison of experimental and simulated dissolution profiles and floatation behavior. Eur. J. Pharm. Sci. 2014, 58, 34-43. [CrossRef]

35. Eberle, V.A.; Häring, A.; Schoelkopf, J.; Gane, P.A.C.; Huwyler, J.; Puchkov, M.; Eberle, V.A.; Häring, A.; Schoelkopf, J.; Patrick, A.C.; et al. In silico and in vitro methods to optimize the performance of experimental gastroretentive floating mini-tablets. Drug Dev. Ind. Pharm. 2015, 42, 808-817. [CrossRef]

36. Food and Drug Administration (FDA). Centre for Drug Evaluation and Research (CDER) Guidance for Industry Guidance for Industry Dissolution Testing of Immediate. FDA 1997, 4, 15-22. 
37. Hwang, K.M.; Nguyen, T.T.; Seok, S.H.; Jo, H.I.; Cho, C.H.; Hwang, K.M.; Kim, J.Y.; Park, C.W.; Rhee, Y.S.; Park, E.S. Swellable and porous bilayer tablet for gastroretentive drug delivery: Preparation and in vitro-in vivo evaluation. Int. J. Pharm. 2019, 572, 118783. [CrossRef] [PubMed]

38. Krausbauer, E.; Puchkov, M.; Betz, G.; Leuenberger, H. Rational estimation of the optimum amount of non-fibrous disintegrant applying percolation theory for binary fast disintegrating formulation. J. Pharm. Sci. 2008, 97, 529-541. [CrossRef] [PubMed]

(C) 2020 by the authors. Licensee MDPI, Basel, Switzerland. This article is an open access article distributed under the terms and conditions of the Creative Commons Attribution (CC BY) license (http://creativecommons.org/licenses/by/4.0/). 